\title{
Evidence of a functional reorganization in the auditory dorsal stream following unilateral hearing loss
}

\author{
Nicolas Vannson ${ }^{\text {a, b, e,*, Kuzma Strelnikov }}{ }^{\text {d }}$, Chris J. James ${ }^{e}$, Olivier Deguine ${ }^{\text {a, b, c }}$, \\ Pascal Barone ${ }^{\text {a, b, } 1}$, Mathieu Marx ${ }^{\text {a, b, c, } 1}$ \\ ${ }^{a}$ Brain and Cognition Research Centre, University of Toulouse Paul Sabatier, Toulouse, France \\ ${ }^{\mathrm{b}}$ Brain and Cognition Research Centre, CNRS-UMR, 5549, Toulouse, France \\ ${ }^{\mathrm{c}}$ Service d'Otologie, Otoneurologie et ORL pédiatrique, Hopital Pierre-Paul Riquet, CHU Toulouse Purpan, France \\ ${ }^{\mathrm{d}}$ Hopital Pierre-Paul Riquet, CHU Toulouse Purpan, France \\ ${ }^{\mathrm{e}}$ Cochlear France SAS, Toulouse, France
}

\section{A R T I C LEIN F O}

\section{Keyword:}

Spatial hearing

Localization

Unilateral hearing loss

Dorsal/ventral auditory streams

\begin{abstract}
A B S T R A C T
Unilateral hearing loss (UHL) generates a disruption of binaural hearing mechanisms, which impairs sound localization and speech understanding in noisy environments. We conducted an original study using fMRI and psychoacoustic assessments to investigate the relationships between the extent of cortical reorganization across the auditory areas for UHL patients, the severity of unilateral hearing loss, and the deficit in binaural abilities. Twenty-eight volunteers (14 UHL patients) were recruited (twenty-two females and six males). The brain imaging analysis demonstrated that UHL induces a shift in aural dominance favoring the better ear, with a cortical reorganization located in the non-primary auditory areas, ipsilateral (same side) to the better ear. This reorganization is correlated not only to the hearing loss severity but also to spatial localization abilities. A regression analysis between brain activity and patient's performance clearly showed that the spatial hearing deficit was linked to a functional alteration of the posterior auditory areas known to process spatial hearing. Altogether, our study reveals that UHL alters the dorsal auditory stream, which is deleterious to spatial hearing.
\end{abstract}

\section{Introduction}

The ability to locate sound sources in the environment facilitates inter-individual communication and is a key skill for self-protection. Sound localization deficits result from binaural hearing alterations and are usually accompanied by other hearing impairments (e.g., poor speech recognition in noise), which may lead to poorer quality of life (Heinrich et al., 2019; Vannson et al., 2015). Based on connectivity (Kaas and Hackett, 1998) and electrophysiological (Rauschecker and Tian, 2000) studies in nonhuman primates, several authors have suggested that spatial hearing is supported by a dorsal where stream, in opposition to a ventrally directed auditory stream involved in sound identification (Arnott et al., 2004; Rauschecker and Scott, 2009; van der Heijden et al., 2019). In humans, lesion studies support the duality of segregated networks for spatial and nonspatial auditory information processing, extending the segregation to the parieto-frontal and temporal lobes (Adriani et al., 2003; Clarke et al., 2002; Zündorf et al.,
2016). Similarly, focal inactivation of activity targeting either the anterior or posterior auditory cortex impairs respectively, identification or localization performance (Ahveninen et al., 2013). Lastly, brain imaging studies support the notion of a dual pathway, and it is now evident that in the early stages, the planum temporale is more specifically modulated by sound position than the primary (core) auditory cortex (PAC) (van der Heijden et al., 2018; Van der Zwaag et al., 2011).

In the case of unilateral hearing loss (UHL), binaural processing is disrupted, leading to poorer localization (Abel and Lam, 2008) and speech recognition performance in noisy environments (Bronkhorst and Plomp, 1989). At the brain level, a small number of studies have explored the functional reorganization that takes place in adults after UHL. As neuronal sensitivity to binaural disparities in terms of time and level is present all along the central auditory pathway, from the lower brainstem to the auditory cortex (Grothe et al., 2010; Sadoun et al., 2020), UHL would be expected to impact binaural integration at all stages. Interestingly, functional reorganization has been observed at the

\footnotetext{
* Corresponding author. CNRS CERCO UMR 5549 CHU Toulouse Purpan - Pavillon Baudot BP 25202 31052, Toulouse Cedex, France.

E-mail address: vannson.nicolas@gmail.com (N. Vannson).

${ }^{1}$ Pascal Barone and Mathieu Marx equally contributed.
} 
level of the auditory cortex after acquired UHL but seems to be absent at subcortical levels (Langers et al., 2005), a result that could be explained by the weak spatial resolution of deep brain structures (subcortical structures such as the inferior colliculus for instance that are hardly defined with fMRI (Schonwiesner et al., 2007). Human brain imaging studies in UHL thus report a decrease in normal interhemispheric asymmetry in cortical auditory activation after stimulation of the preserved ear. Whereas monaural stimulation induces a more pronounced level of activity in the contralateral (opposite) auditory cortex in normal hearing persons, more bilateral activation has been demonstrated in cases of UHL, using either fMRI (Burton et al., 2012; Scheffler et al., 1998; Suzuki et al., 2002), or EEG/MEG (Hanss et al., 2009; Vasama and Mäkelä, 1995). However, contradictory results make it unclear whether the functional subdivisions of the auditory cortex are differently affected by acquired UHL (Hanss et al., 2009; Scheffler et al., 1998). The disruption of binaural processes should predominantly impact the dorsal auditory stream, but this has not been explored. Furthermore, several reports have indicated that patients with congenital unilateral deafness (Slattery III \& Middlebrooks, 1994) exhibit close to normal localization performances. This suggests that patients can develop adaptive compensatory strategies, which should have a specific signature in brain activity. Altogether, many questions remain to be resolved, concerning the brain plasticity mechanisms that may occur in patients with acquired UHL and their putative impact on behavioral performances.

The psychoacoustic effects of acquired unilateral hearing loss can be evaluated within a normal hearing population using a plug in one ear. Under these conditions, the deficit for spatial hearing has been demonstrated in several studies (for instance Van Wanrooij and Van Opstal, 2007). Still, performances for spatial hearing remain poorer than that observed following long-term hearing loss (Alzaher et al., in prep; Kumpik et al., 2010; Vannson et al., 2017); where a behavioral adaptation has occurred. In addition, no evidence of immediate plastic reorganization has been described in such acute deprivation (Van Wanrooij and Van Opstal, 2007). The behavioral and cortical consequences of postlingual acquired unilateral hearing loss should be distinguished from those occurring after congenital unilateral hearing loss. In the latter case, the development is characterized by the presence of different sensitive periods, expressed as a susceptibility to behaviorally adapt to sensory privation as well as a potential of brain plasticity that decreases with age (A. Kral, 2013; Syka, 2002).

We conducted a study that aimed at investigating the relationships between UHL severity, the resulting deficit in spatial hearing, and the extent of cortical reorganization across auditory areas. Our results clearly demonstrate that a unilateral deafness results in a sound localization deficit that is related to a functional shift towards ipsilateral aural dominance. The core of the auditory cortex appears relatively preserved but UHL impacts specifically the posterior auditory areas of the dorsal pathway involved in spatial hearing while the ventral temporal stream areas maintain their normal implication in processing non-spatial acoustical information.

\section{Materials and methods}

This study was approved by the local institutional review board (Comité de Protection des Personnes du Sud Ouest et Outre-Mer IV; no. CPP14-021/2014-A00498-39) in accordance with the Declaration of Helsinki.

\subsection{Participants}

Fourteen right-handed (according to the Edinburgh Handedness Inventory; Oldfield, 1971) adults ( 11 females; median age $=39$ years, $S D$ $=\quad 13,06)$ and native French speakers with an acquired UHL were recruited. Ten of them had a better right ear (left UHL), and four had a

better-left ear (right UHL). The only inclusion criterion was UHL established by pure-tone audiometry, ranging from mild to total in order to ensure a representative sample. All participants with UHL had normal contralateral hearing thresholds (i.e. below $20 \mathrm{~dB}$ HL between $125 \mathrm{~Hz}$ and $8 \mathrm{kHz}$ ). Mean pure-tone average (PTA) was $87 \mathrm{~dB} \mathrm{HL}$, covering a range of 37 to over $120 \mathrm{~dB}$. The main characteristics of this population can be found in Table 1 .

Furthermore, 14 age- and sex-matched normal-hearing subjects (NHS) were recruited with a comparable median age (36.5 years, $S D=$ 14 ; bootstrap confidence intervals, $p>0.05$ ) and with hearing thresholds within the normal range for both ears. All participants underwent psychoacoustic tests and fMRI scans. The fMRI data of one participant with UHL (subject 11) were excluded because of technical problems during the fMRI acquisition.

\subsection{Psychoacoustic procedures}

Clinical pure-tone and speech audiometry was carried out with a GN Otometrics Madsen Itera 2 audiometer with TDH-39 headphones. Binaural hearing was assessed in sound field in a calibrated soundproof booth. Binaural hearing encompassed the French Matrix test (FrMatrix; Jansen et al., 2012) for speech-in-noise recognition and a clinical set-up sound localization test routinely used in the ENT department (Vannson et al., 2015, 2017).

\subsubsection{Speech-in-noise recognition test: FrMatrix}

The FrMatrix is an adaptive and standardized test for speech-in-noise recognition that features closed-set sentences. Each sentence has the same syntactic structure: name - verb - number - object - color. For instance: "Felix draws six blue bikes". The background noise is a stationary long-term average speech spectrum noise that was generated by superimposing all 280 sentences Speech and noise were generated by an IBM PC running the OMA software and presented by three loudspeakers and an amplifier (Studio Lab, SLB sat 200). Speech was set at 65 dB SPL, and competing noise was adjusted to obtain a signal-to-noise ratio of $50 \%$ (SNR50) for correct recognition. SNR50 was measured in three sound field listening conditions: i) the dichotic condition with the signal presented to the poorer ear (PE) and the noise to the contralateral, better ear (BE); ii) the diotic condition with both signal and noise collocated and presented from the loudspeaker located in front of the subject; and iii) the reverse-dichotic condition with the signal presented to the better ear loudspeaker and the noise to the poorer ear loudspeaker. Subjects were asked to repeat any words they heard.

\subsubsection{Sound localization test}

Localization ability was assessed in the ENT department with a horizontal array of 7 loudspeakers and an amplifier located at intervals of $30^{\circ}$ from $-90^{\circ}$ to $90^{\circ}$ in a frontal semicircle diameter of $1.2 \mathrm{~m}$ at the subject's head level. Localization stimulus was made up of two $150 \mathrm{msec}$ white Gaussian noise from $20 \mathrm{~Hz}$ to $20 \mathrm{kHz}$ with a $0.05 \mathrm{msec}$ ramp. A silence of $150 \mathrm{msec}$ was encompassed between the two noises for total stimulus duration of $450 \mathrm{msec}$. This stimulus was similar to the one used by Slattery and Middlebrooks (Slattery III \& Middlebrooks, 1994) and was presented 63 times ( 9 presentations per loudspeaker) with a period of $2 \mathrm{~s}$ silence between each presentation to allow the subjects to give orally the loudspeaker location. Localization ability was measured with the standard root-mean-square (RMS) errors. The examiner was in the room with the patient to control that he/she keeps his/her head in front of the speaker $1\left(0^{\circ}\right)$ throughout the experiment. The patient had a sheet of paper with the position and the number of all speakers to help $\mathrm{him} / \mathrm{her}$. If the patient was not able to point out to a loudspeaker, his/her answer was marked as "No answer". For each patient, we had at least 60 out of 63 valid responses (less than $5 \%$ missing data). 
Table 1

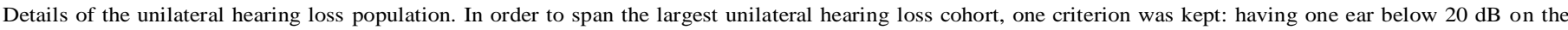

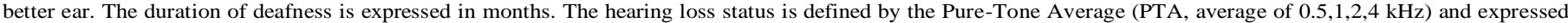

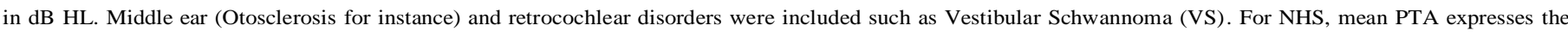
average PTA of the right and left ear PTA.

\begin{tabular}{|c|c|c|c|c|c|c|c|c|c|}
\hline Subjects & Sex & Age (years) & Etiology & Poorer ear & Duration (months) & $\begin{array}{l}\text { PTA } \\
\text { Better ear }\end{array}$ & $\begin{array}{l}\text { PTA } \\
\text { Poorer ear }\end{array}$ & $\begin{array}{l}\text { NHS } \\
\text { Age (years) }\end{array}$ & NHS mean PTA \\
\hline 1 & $\mathrm{~F}$ & 28 & Chronic otitis sequelae & Left & 12 & 10 & 37.5 & 29 & 9.375 \\
\hline 2 & $\mathrm{~F}$ & 46 & Otosclerosis & Right & 96 & 3.75 & 26.25 & 47 & 3.125 \\
\hline 3 & F & 22 & Sudden hearing loss & Left & 204 & 12.5 & 120 & 24 & 6.25 \\
\hline 4 & $\mathrm{~F}$ & 61 & VS stage III & Left & 72 & 16.25 & 120 & 65 & 11.875 \\
\hline 5 & F & 35 & Otosclerosis & Left & 48 & 8.75 & 45 & 37 & 5.625 \\
\hline 6 & F & 31 & Perilymph fistula & Right & 12 & 13.75 & 120 & 34 & 8.125 \\
\hline 7 & F & 58 & Cholesteatoma & Left & 192 & 15 & 65 & 63 & 20 \\
\hline 8 & F & 39 & Otosclerosis & Left & 240 & 20 & 46.25 & 28 & 2.5 \\
\hline 9 & $\mathrm{~F}$ & 56 & Cholesteatoma & Left & 120 & 16.25 & 48.75 & 53 & 15.625 \\
\hline 10 & $\mathrm{~F}$ & 35 & Cholesteatoma & Left & 384 & 16.25 & 57.5 & 33 & 11.25 \\
\hline 11 & $\mathrm{~F}$ & 42 & Chronic otitis & Right & 84 & 20 & 75 & 43 & 8.125 \\
\hline 12 & M & 23 & Temporal bone trauma & Left & 12 & 3.75 & 43.75 & 26 & 1.875 \\
\hline 13 & M & 50 & Tympanic perforation & Left & 24 & 11.25 & 35 & 59 & 6.875 \\
\hline 14 & M & 38 & Meniere Syndrome & Right & 350 & 15 & 120 & 36 & 6.25 \\
\hline
\end{tabular}

\section{MRI experiment}

\subsection{Stimuli}

Six voices and six nonvoices stimuli (Belin et al., 2000) were used as ecological stimuli to activate the auditory system. All stimuli lasted $0.5 \mathrm{~s}$

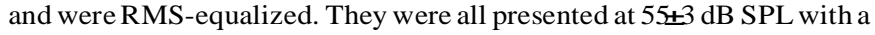
pneumatic system that uses earplugs (NordicNeurolab®). This sound level was chosen to avoid any interaural transcranial transfer function, which may occur above $60 \mathrm{~dB}$ (Scheffler et al., 1998), and was measured at the earplug extremity with a ROLINE® (RO-135) sound-level meter.

Each stimulus was presented 5 times (for instance: $5 \mathrm{X}$ voice $1,5 \mathrm{X}$ voice $2, \ldots$ ) per condition (binaural, left and right). The impact of the category of the stimulus (voice vs non-voice) was assessed. Thus, a total of 30 stimuli for voice and non-voice per condition were presented.

Because there was no significant difference between voice and nonvoices for the NHS (bootstrap, $\mathrm{p}>0.05$ ), all the stimuli were pooled for further analysis. We ended up with 60 stimuli (trials) per condition so a total of 180 trials and 30 silences trials as baseline. This protocol was previously validated during a pilot session to insure a significant brain activation without generating fatigue for the patient.

3.2. Task

Participants were each asked to perform a two-alternative forced choice (2FAC) discrimination task by pressing one button when they heard a voice and a different button when they heard a non-voice stimulus. Participants were asked to keep their eyes open to maintain their attention and to follow the instructions (Button 1: "voice"; Button 2: "non-voice") on the screen. All stimuli and responses were controlled using Presentation ${ }^{\circledR}$ (Neurobehavioral Systems).

\subsection{Paradigm}

Similarly to Burton et al. (2012), our paradigm was designed basing on the previous work done by Belin et al., in 1999 where the time course of the BOLD (Blood Oxygen level-dependent) response within the left and right primary auditory cortex was measured using a sparse temporal sampling method. To measure the full BOLD response of the voice and non-voice stimulus presented randomly and separately, our design encompassed $5 \mathrm{~s}$ of silence after EPI (Echo-Planar Imaging) and $3 \mathrm{~s}$ of silence again after the stimulus presentation in order to record the full peak of the BOLD response.

\subsection{Image acquisition and fMRI analysis}

BOLD and anatomical images were acquired on a 3 T Philips ACHIEVA X-series MRI scanner at the MRI platform of INSERM U1214 ToNIC. As in Belin et al. (1999) we used a sparse temporal sampling method with a 5-s silence after the start of MRI acquisition and before the stimulus presentation, and a 3-s silence after the stimulus presentation in order to record the peak BOLD response. Anatomical images were first recorded with the following parameters: T1 gradient-echo, TR of $8.1 \mathrm{~ms}$, TE of $3.7 \mathrm{~ms}$, flip angle of $8^{\circ}$, Field of View of $240 \times 240 \mathrm{~mm}$ and a $1 \times 1 \times 1 \mathrm{~mm}$ resolution. 170 anatomical images were recorded in total. As for the BOLD signal images, the T2* EPI had the following parameters: TR $=10,500 \mathrm{~ms}, \mathrm{TE}=30 \mathrm{~ms}$, TA of $2085 \mathrm{~ms}$, flip angle of $90^{\circ}, \mathrm{FOV}=240 \times 240 \mathrm{~mm}$, voxel size $3 \times 3 \times 3 \mathrm{~mm}$ and matrix size $80 \times$ 80 pixels. 40 slices were acquired per volume and 43 vol were acquired per run for a total of 5 runs forming $215 \mathrm{vol}$ in total. The first dummy volume of each session was removed due $T_{1}$ magnetization equilibrium. The duration of the functional MRI was 8 min per run, and the total experiment lasted about $50 \mathrm{~min}$.

All MRI images were analyzed with SPM8. Images, then realigned within each subject, normalized towards the MNI152 template and finally smoothed by a Gaussian filter with a FWHM of $8 \mathrm{~mm}$. We used a one-way ANOVA with Condition as factor to compare each voxel between conditions. This statistical method is equivalent to a general linear model (Belin et al., 1999; Weeks et al., 2000). The global signal was normalized within and between subjects before comparison. We used two maximum probabilistic maps as regions of interest (ROIs) to avoid misclassified active voxels (Eickhoff et al., 2005). These regions have been cytoarchitectonically defined in humans (Morosan et al., 2001, 2005): the primary auditory cortex (PAC), encompassing Te1.0, $\mathrm{Te} 1.1$ and Te1.2, and the non-primary auditory cortex (NPAC) comprising Te3.0 (Fig. 1). Both ROIs were implemented in the SPM Anatomy Toolbox (Eickhoff et al., 2005). These two ROIs were thus employed to extract the extent of significant activated voxels (at $\mathrm{t}=$ $2.34, \mathrm{P}=0.01$ ) and the average intensity (percent of BOLD signal change) over each ROI (Jamison et al., 2006).

\subsection{Weighted indices of brain activity}

To carry out an overall assessment of auditory activation during the task, we computed a composite measure of brain activity that takes into account both the mean intensity (Int) and extent (number of voxels, Nvox) of activation in the ROI (Jamison et al., 2006). This index reduced the risk of floor or ceiling effects (Stefanatos et al., 2008). This measure of brain activity was further applied to two different indices. 


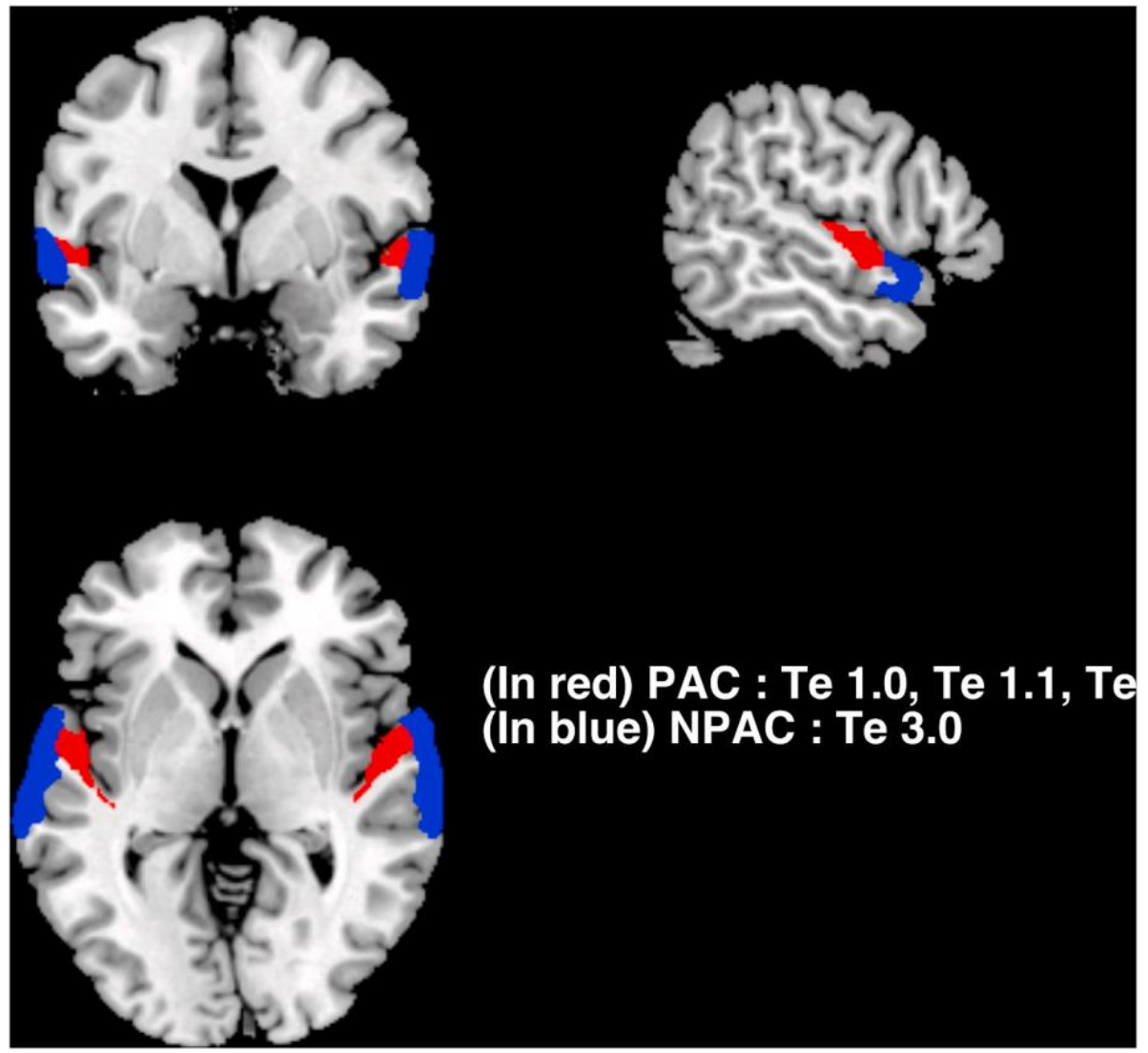

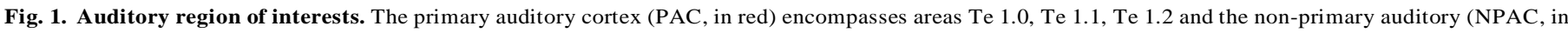
blue) the Te 3.0 region. (For interpretation of the references to color in this figure legend, the reader is referred to the Web version of this article.)

First, hemispheric lateralization was computed using the weighted laterality index (WLI; Stefanatos et al., 2008) and based on the composite activity computation:

$\mathrm{WLI}=($ left $($ Nvox $\mathrm{x}$ Int $)-\operatorname{right}($ Nvox $\mathrm{x}$ Int $)) /($ left $($ Nvox $\mathrm{x}$ Int $) \quad+$ right (Nvox x Int))

A WLI close to 0 means symmetrical brain activation. When WLI tends towards either $\#$ or 1 , it reflects asymmetrical brain activation towards the left or right hemisphere.

Second, we computed a binaural integration index (BII) using the composite activity, in order to assess how the auditory cortex responded to a sound that was simultaneously presented to both ears (BIN), compared with the summed activity obtained after separate stimulation of each ear $(\mathrm{R}+\mathrm{L})$ :

\section{$\mathrm{BII}=\mathrm{BIN} /(R+L)$}

A BII below 1 corresponds to a suppression mechanism induced by binaural integration. By contrast, a BII above 1 reflects facilitation processes.

\subsection{Experimental design and statistical analysis}

All the analyses were implemented in MATLAB r2014b and R (3.3.3). For all the behavioral measures, such as localization, speech-in-noise recognition and hit rate for voice vs non-voice distinction, mean differences between participants with UHL and NHS were compared using a nonparametric, bootstrap technique to generate $95 \%$ confidence intervals $(10,000$ samples; bias corrected and accelerated confidence intervals; alpha $\oplus .05$; Carpenter and Bithell, 2000). In addition, this bootstrap technique was also applied to compare groups on brain activity indices (WLI and BII). Finally, we performed a regression analysis between behavioral measures and brain activation, and all significant clusters ( $p<0.05$, with familywise error correction, FWE) were identified with probabilistic cytoarchitectonic maps (Eickhoff et al., 2005) and the Harvard/Oxford Atlas in MRIcron (2016).

\section{Results}

\subsection{Binaural hearing assessment}

We assessed binaural hearing abilities of all participants using a localization test and the FrMatrix (Fig. 2A and B). Average performance of participants with UHL on these two tasks clearly revealed a substantial deterioration in their binaural hearing processing, as their mean

RMS error for localization was $27.70^{\circ}(S D=28.33)$, which was considerably poorer (bootstrap, alpha $=0.05)$ than for NHS $\left(1.19^{\circ}, S D\right.$ $=1.24$ ) (Fig. 2A, right). The deficit was observed for all azimuths but was especially important for sounds on the side of the poor ear, for which most RMS values exceeded $40^{\circ}$ (Fig. 2A, left).

For speech-in-noise recognition (FrMatrix; Fig. 2B), SNR50s did not differ between dichotic and reverse-dichotic conditions for NHS (bootstrap, alpha $0 € 5$ ), and were therefore pooled. Mean SNR50 was $-14.50 \mathrm{~dB}(S D=1.39)$ in the dichotic and reverse-dichotic conditions, and $5.37 \mathrm{~dB}(S D=.20)$ in the diotic condition. Compared with the NHS group, participants with UHL scored lower in each condition (bootstrap, alpha $\theta_{05}$; Fig. 2B). Their mean SNR50s were $-2.00 \mathrm{~dB}$ $(S D=5.31)$ in the dichotic condition, $-3.14 \mathrm{~dB}(S D=1.19)$ in the diotic condition, and $-10.62 \mathrm{~dB}(S D=2.62)$ in the reverse-dichotic condition.

In addition, the degree of UHL, as measured by the poorer ear PTA, was significantly correlated with speech-in-noise recognition (dichotic SNR50s, rho $=0.80, p<0.001)$ and horizontal sound localization abilities (mean RMS values, rho $=0.75, p=0.002$ ). In the UHL group, 
A

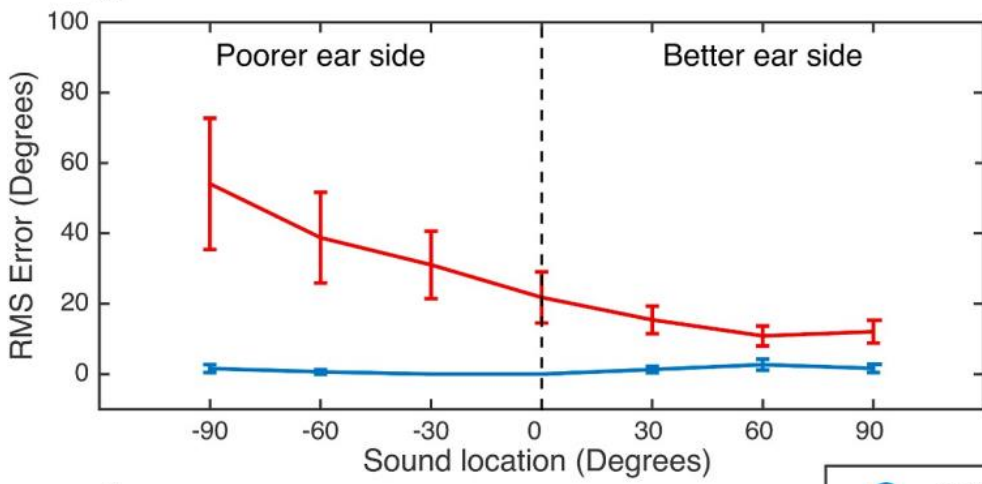

Average

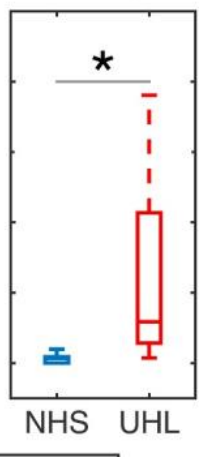

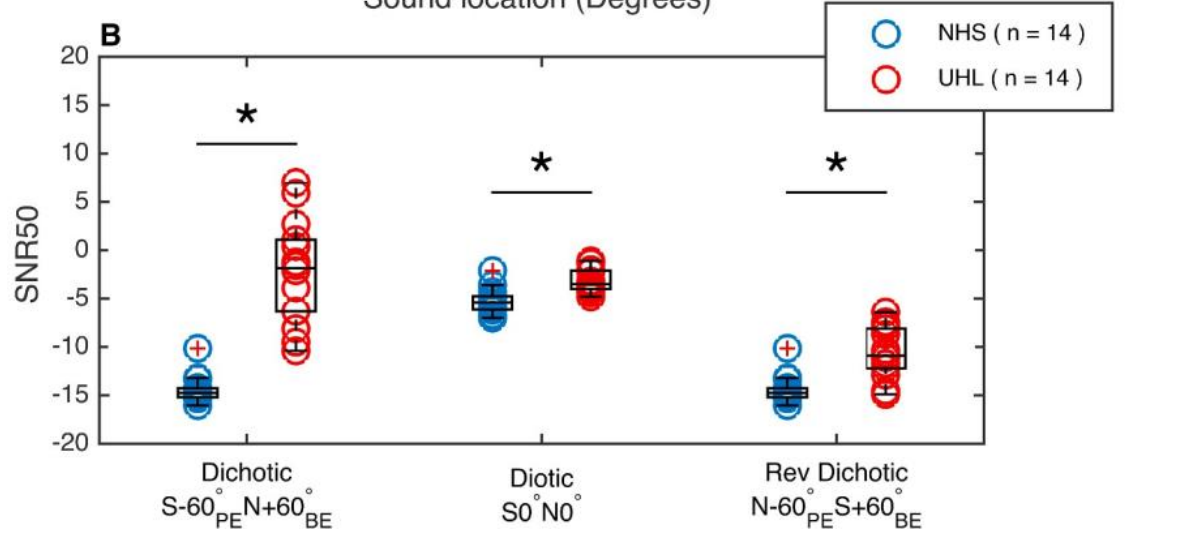

Fig. 2. Binaural hearing evaluation. (A) Sound localization performances of NHS (blue) and UHL participants (red). RMS errors $(+/-$ SEM) are displayed according to the sound position (left panel) or averaged across speakers (right panel). Participants with UHL exhibited a strong deficit, reflected by a higher RMS compared with NHS, especially on the side of the deaf ear. (B) FrMatrix test. The performances of the NHS (blue) and participants with UHL (red) on speech-in-noise recognition are expressed as the speech recognition score corresponding to the $50 \%$ correct threshold (see text) in the three different presentation conditions. $\mathrm{PE}=$ poorer ear; $\mathrm{BE}=$ better ear; $\mathrm{S}=$ speech; $\mathrm{N}=$ noise. In all the panels, asterisks indicate a significant difference based on bootstrap 95\% confidence intervals $(\alpha=0.05)$. (For interpretation of the references to color in this figure legend, the reader is referred to the Web version of this article.) poor localization abilities were also significantly associated with a deficit in speech-in-noise recognition in the dichotic condition (rho $=$ $0.69, p=0.006)$.

In conclusion, participants with UHL exhibited a significant deficit in integrating binaural cues, the magnitude of this deficit being related to UHL severity, in line with previous studies (Vannson et al., 2015, 2017).

\section{Sound categorization task}

Both groups performed a voice/non-voice discrimination task (Massida et al., 2011) in the scanner, in three listening conditions (right, left, and binaural). The hit rate for participants with UHL in the binaural listening condition (voices and non-voices summed) was similar to that observed for NHS $(93.66 \%, S \oplus 6.13$ vs. 95.80\%, SE 3.77, bootstrap, alpha $\otimes_{05}$ ), reflecting a near-to-normal BE performance level. As expected, poorer ear performances of participants with UHL were low (16.66\%, SD 28.72), with a tendency to systematically classify the stimulus as a non-voice one (Sup Fig. 1).

\subsection{Neurobiological investigation by fMRI}

\subsubsection{BOLD activation at whole-brain level}

Brain activation was induced by environmental sounds delivered to either one ear or both ears. As responses to voice and non-voice stimuli did not differ significantly within the NHS group (bootstrap, alpha = 0.05 ), both were summed. Besides, the impact of the side of unilateral hearing loss on cortical reorganization is still controversial. Some EEG studies (Hanss et al., 2009; Khosla et al., 2003; Po-Hung Li et al., 2003) showed a clear impact of the deafness side, with more plasticity in case of left hearing loss. In contrast, this right ear/left ear effect was not found in several fMRI studies (Bilecen et al., 2000; Scheffler et al., 1998), which showed similar patterns of reorganization whatever the affected side. Therefore, to avoid stirring misleading conclusions we decided to split our population into two subgroups: good right better ear $(\mathrm{n}=10)$ and 3-left better ear. In the Results section, we focus on the left UHL subgroup, as it contained more participants. The data for the right UHL subgroup are provided in the Supplementary Information (Sup Fig. 3 and 4).

Overall, in the NHS group, monaural stimulation led to greater contralateral brain activation, whatever the presentation side, whereas binaural stimulation induced symmetrical brain activation (Fig. 3, left). These results were observed at the whole-brain level, but also at the level of the two ROIs within the auditory cortex.

In the UHL group with right BE (Fig. 3, right), left PE stimulation induced low but significant activation of the auditory cortices, owing to the broad range of hearing loss severity. Right BE stimulation induced

strong contralateral activation (left hemisphere), as in NHS, but substantial activity was also observed in the right, ipsilateral auditory areas. This trend towards a pattern of bilateral activation after BE stimulation was also observed when the BE and PE were simultaneously stimulated.

To gain better insight into the mechanisms of brain reorganization after UHL, we conducted separate analyses on the PAC and NPAC.

\subsubsection{Composite auditory brain activity in unilateral hearing loss}

The levels of activity induced by the stimuli were first analyzed in the PAC and NPAC of participants with UHL. As at the whole-brain level (Fig. 3), the level of activity induced after left-ear stimulation was extremely weak in both left and right PAC and NPAC (Sup Fig. 2). Rightear and binaural stimulation both led to greater activity in the left (i.e., contralateral to the BE) PAC, but the levels of activity appeared to be different in the NPAC, with a trend towards greater mean activity in the right hemisphere (i.e., side ipsilateral to the $\mathrm{BE}$ ).

In order to provide a better account of individual variability than mean brain activity, we analyzed how the binaural integration and lateralization indices were modified in patients with UHL compared with NHS.

\subsubsection{Binaural interaction revealed by the binaural integration index (BII)}

Binaural integration mechanisms occur when the BII is significantly different from 1.0 (Fig. 4). In the NHS group, whatever the hemisphere, 

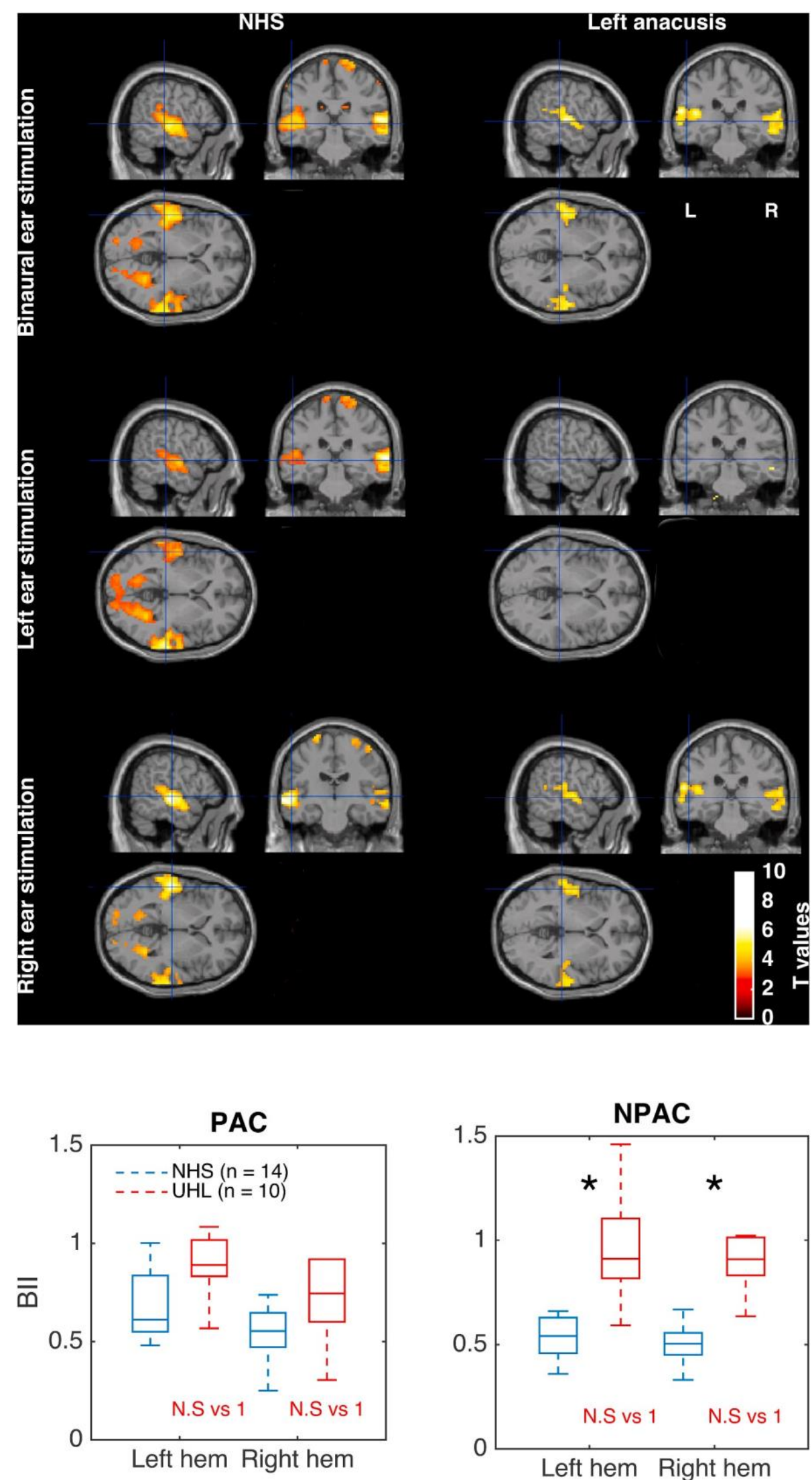

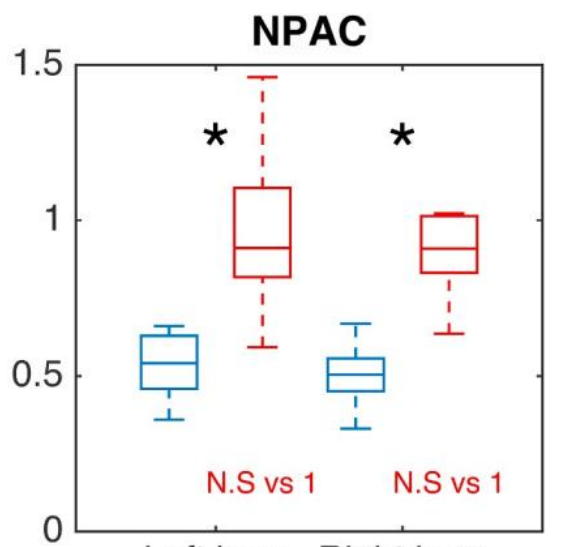

Left hem Right hem
Fig. 3. BOLD activation at the whole-brain level. Brain activity for NHS (left column) and participants with a left poorer ear (left UHL, right column). In both groups, monaural stimulation induced bilateral activation in auditory areas with a tendency in NHS towards more contralateral activity. In UHL, deaf ear stimulation did not elicit a significant response, while stimulation of the intact ear appeared to similarly activate both left and right cortices. For the purposes of display, sagittal, coronal and horizontal views are illustrated at $\mathrm{p}$ uncorrected $=0.001$.
Fig. 4. Binaural integration index. This index (BII) was obtained with a composite computation of signal intensity and number of activated voxels. BII values are presented for NHS (blue) and participants with UHL (red), separately for the left and right hemispheres, and in both cortical areas (PAC and NPAC). A BII below +1.0 indicates that the activity observed following binaural stimulation was lower that the summed activity resulting from each monaural stimulation. This suppression mechanism was observed in NHS in both the PAC and NPAC. In participants with UHL, the suppression mechanisms were reduced in the PAC and absent in the NPAC. The black asterisks show a significant difference between NHS and UHL. The Ns vs. 1 (red) indicates that the BII values for participants with UHL did not differ from 1 , based on bootstrap $95 \%$ confidence intervals $(\alpha=0.05)$. (For interpretation of the references to color in this figure legend, the reader is referred to the Web version of this article.) 
mean BII values were significantly below 1.0 (bootstrap, alpha $=0.05$ ) within the PAC (left hem $=0.79(\mathrm{SD}=0.48)$; right hem $=0.58(\mathrm{SD}=$ $0.31))$ and NPAC $($ left hem $=0.60(\mathrm{SD}=0.31)$; right hem $=0.50(\mathrm{SD}=$ 0.10)) reflecting a binaural suppression mechanism, as the composite activation level induced by the simultaneous stimulation of both ears was significantly lower than the sum of individual activations. In the PAC and NPAC, mean BII values were statistically similar, but there was a trend toward greater binaural suppression in the NPAC than in the PAC.

In the UHL group, none of the mean BII values differed significantly from 1 (bootstrap, alpha $=0.05)$ in the PAC (left hem $=0.86(\mathrm{SD}=$ $0.29)$; right hem $=0.87(\mathrm{SD}=0.60))$ and NPAC $($ left hem $=0.97(\mathrm{SD}=$ $0.27)$; right hem $=0.97(\mathrm{SD}=0.39)$ ), whatever the hemisphere. These results can be interpreted as a loss of binaural integration, which was particularly apparent within the NPAC, with mean BII values significantly higher and closer to values of 1 , than those observed in NHS. In the PAC, mean BII values did not differ significantly from those observed in the NHS group.

\subsubsection{Effect of unilateral deafness on aural dominance as revealed by the weighted laterality index}

A WLI close to+1.0 or -1.0 indicates a stronger contralateral left or contralateral right dominance. A WLI close to 0 corresponds to an equivalent level of activity across the two hemispheres.

In the NHS group, monaural stimulation (left or right ear; Fig. 5C) induced a strong contralateral dominance within the PAC, expressed by mean WLI values of -0.32 (left stimulation) and 0.47 (right stimulation), both significantly different from zero (bootstrap, alph 0.05) The mean absolute WLI values after right- or left-ear stimulation were comparable (bootstrap, alpha $=.05$ ), reflecting an equivalent contralateral dominance whatever the stimulated ear. In addition, binaural stimulation led to significant (bootstrap, alpha 0.05) hemispheric lateralization that favored the left hemisphere (WH $\oplus .25)$. This was nevertheless smaller than the strong leftward lateralization observed following right-ear stimulation.

Within the left UHL subgroup (Fig. 5A), we observed a clear reorganization of brain activity, reflected by major WLI modifications However, these changes in the lateralization of brain activity depended on the cortical auditory area. Within the PAC, both binaural and rightear stimulation resulted in a similar pattern to that observed in NHS, with greater activation in the left hemisphere (bootstrap, alph 0.05), contralateral to the BE. The WLI following right-ear stimulation was comparable to that in the NHS groupt ( 0.57 v $0.61,1=0.425)$. In addition, after binaural stimulation, the UHL group had a slightly, but not significantly ( $\mathrm{p} \oplus .062)$, higher WLI than NHS, corresponding to greater left lateralization. This left lateralization probably resulted from the absence of binaural suppression in the left PAC, as previously described (see Fig. 4), and from the activation generated by the BE. This was confirmed by the strong individual correlation between the indices for the binaural and right-ear conditions ( $\mathrm{r}$ \&80, $\mathrm{p}<0.001)$.

In the NHS group, the pattern of activation was weaker in the NPAC (Fig. 5D) than in the PAC, but was similar following the monaural stimulation, with a substantial contralateral dominance. However, binaural stimulation led to symmetrical interhemispheric activation, with a mean WLI that did not differ from zero (WEI+0.09; bootstrap, alpha $\oplus .05$ ).

In the UHL group, stimulation of the right BE generated a pattern of greater activity in the ipsilateral right hemisphere (Fig. 5B; bootstrap, alpha $\# .05)$. This ipsilateral dominance contrasted with that observed in the NHS group (difference of $-0.47, \mathrm{p}<0.001$ ), characterized by a strong WLI for the contralateral left hemisphere. This reorganization of brain activity in the NPAC was also observed after binaural stimulation, with high level of brain activation (bootstrap, alpha=0.05) in the right hemisphere, ipsilateral to the BE. As for the PAC, the binaural WLI for the right hemisphere mainly resulted from BE stimulation, as a strong correlation was observed between individual WLI values after binaural and right-ear stimulation ( $\mathrm{r} 0-80, \mathrm{p}<0.001$ ).

Brain activation following monaural stimulation of the left $\mathrm{PE}$ is difficult to interpret, owing to the weakness of the overall activation. However, it seems that PE stimulation induced a strong significant shift in brain activation towards the contralateral right PAC, and a potential weak shift towards the left ipsilateral NPAC.

To summarize, our fMRI results revealed that a unilateral hearing deficit affected differently on brain reorganization in the PAC and NPAC. This dichotomy between these two functional areas indicates that binaural integration is strongly altered in the NPAC and, to a less extent,
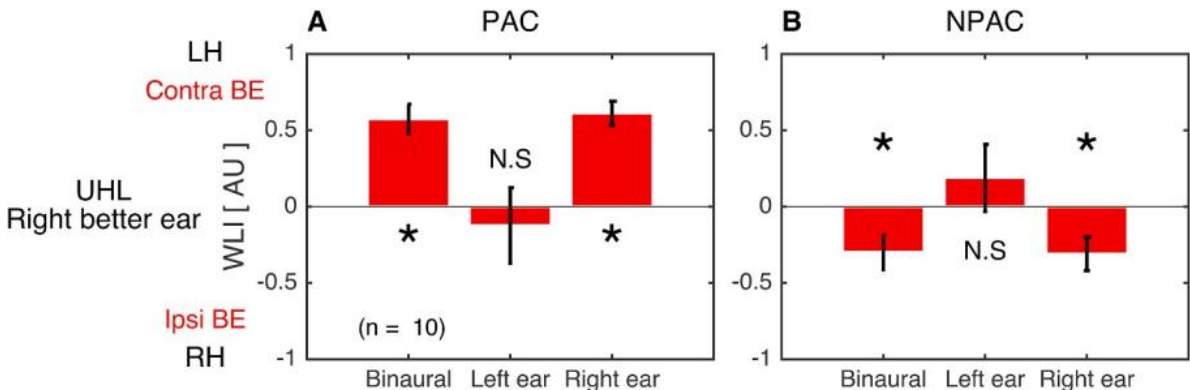

NHS

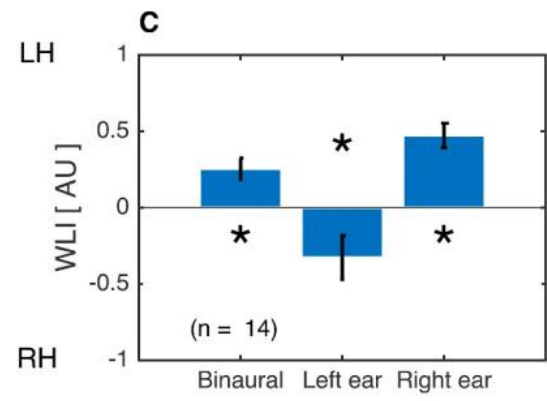

Stimulation side
Fig. 5. Weighted laterality index comparisons between UHL and NHS within the PAC and NPAC. Weighted laterality index (WLI) values for participants with UHL (upper panels) and NHS (lower panels) in both the PAC (left columns) and NPAC (right). WLI values of 0 correspond to a symmetrical pattern of auditory activity, while values towards +1 or -1 correspond to a pattern favoring a specific cortical hemisphere (y-axis), either ipsi- or contralateral to the stimulated ear (x-axis). In both NHS and participants with UHL, monaural stimulation led to a contralateral dominance in the PAC. In participants with UHL, stimulation of the better ear led to a shift toward the ipsilateral hemisphere in the NPAC. The asterisks show a significant difference between 0 and the activation level $(\alpha=0.05$; bootstrap $95 \%$ confidence; NS = no significant) 
in the PAC. When the better ear is stimulated, cortical reorganization is marked by normal contralateral dominance within the PAC and a significant shift of activation towards the ipsilateral hemisphere in the NPAC.

\subsubsection{Relationship between binaural abilities and brain reorganization}

UHL leads to poorer binaural hearing performances and to brain reorganization mechanisms that mainly and significantly affect the NPAC, as expressed by changes in aural dominance (WLI) and binaural interaction (BII). Two approaches were used to further investigate the relationship between cortical reorganization and spatial hearing deficits.

First, we assessed the relationship between hearing performances (PTA, FrMatrix dichotic score and RMS error) and NPAC WLI and BII values. No correlation was found between BII and hearing performances (see Sup Table 1). However, as shown in Fig. 6, the increase in ipsilateral dominance within the right NPAC was associated with poorer sound localization performances (RMS, $\pm-0.79, \mathrm{p}=0.006)$. This strong relationship was controlled by age (partial corr $=\mathrm{r}-0.86,0.003$ ), suggesting that aging had no significant effect (difference between the two correlations $(r=-79$ and $r=-.86$; $\mathrm{z}$-score $=0.41, \mathrm{p}=0.67)$, while hearing loss severity (PTA, partial corr $\neq-0.77, \mathrm{p}=0.016$ ) and duration of hearing loss (partial corr $=-0.79, \mathrm{p}=0.012$ ) where statistically correlated. This result revealed that the stronger the dominance shifts toward the ipsilateral NPAC, the stronger the deficit in horizontal sound localization. No significant correlation was found between the WLI for the right NPAC and the dichotic speech recognition score $(r=0.29$, p $\quad$ 404), but we can hypothesize that the change in aural dominance is also linked to speech-in-noise recognition abilities, as dichotic SNR50 and RMS errors were strongly correlated.

Second, a regression analysis was applied at the whole-brain level between behavioral performances and brain activity after binaural stimulation in both groups. This analysis aimed at identifying the cortical network whose activity accounts for auditory performances concerning either auditory discrimination (voice/non-voice

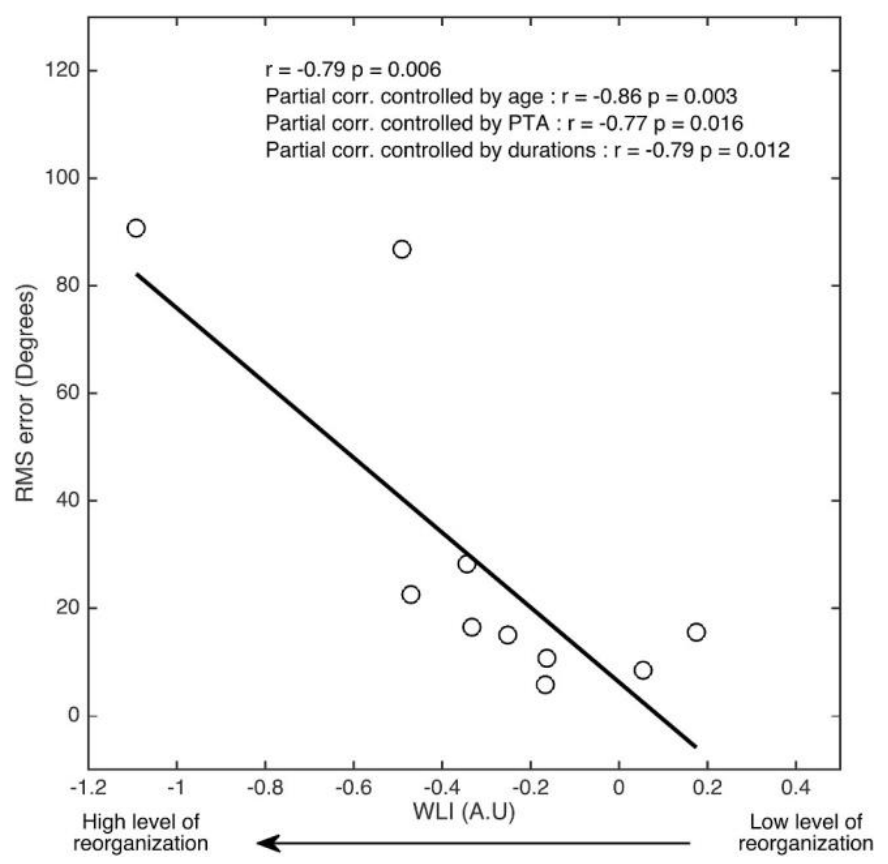

Fig. 6. Relationship between cortical functional reorganization and individual characteristics of participants with UHL. We observed a significant negative correlation between the weighted laterality index (WLI) for the right (better) ear and sound localization performances (RMS errors). This correlation was controlled (significant partial correlations) by age, hearing loss severity and durations of deafness (expressed in months). discrimination) or auditory spatial processing (sound localization).

When the whole-brain regression analysis was applied to discrimination performances (hit rates), we observed similar clusters of activity for both the NHS and UHL groups (Fig. 7). In the NHS group, there was a significant regression of brain activity with sound discrimination scores in the right superior temporal gyrus/sulcus (STG/STS) (p FWE-corrected $<0.001$, peak coordinates $63-251$ ). The regression with hit rates in participants with UHL was also located in the right STG/STS (p FWEcorrected $<0.001$, peak coordinates $63-16-5$ ). No significant difference was found when the NHS and UHL groups were compared on regression with hit rates. Furthermore, the activity extended to anterior auditory areas including the temporal voice area, which is known to be specifically involved in human voice processing (Belin et al., 2000). A large cluster was also present in the parietal and occipital visual regions. These results suggest that the same auditory cortical network is involved in patients with UHL and NHS to achieve normal voice/non-voice discrimination.

Then, a whole-brain regression analysis with the sound location scores (RMS errors) was performed. In NHS, there were significant regressions in the left STG/STS (p FWE-corrected $<0.001$, peak coordinates $-60-19-8$ ) and right STG/STS (p FWE-corrected $<0.03$, peak coordinates $63-254$ ). In participants with UHL, a small amount of residual activity was observed within auditory areas, but no significant regression of brain activity with sound location scores was found. The contrast between NHS and UHL groups for this regression was further explored, revealing that the bilateral STG/STS was significantly involved in sound localization in NHS (Table 2, Fig. 8). These regions are posterior to the PAC, and can be considered to belong to the dorsal auditory pathway (Da Costa et al., 2018). This result clearly demonstrates that the spatial hearing deficit was associated with a deficiency of the dorsal auditory stream in participants with UHL.

Taken together, our results specifically point to the impact of UHL on the functional integrity of the auditory dorsal stream areas, with altered neuronal binaural interactions (probably due to a shift in aural dominance) responsible for the deficit in sound localization.

\subsubsection{Unilateral hearing loss with a left better ear}

Only three participants with a left better ear were included in this study (see Supplementary Information) preventing us from drawing any strong conclusions. At the PAC level, contralateral dominance seemed to be preserved following stimulation of the left BE. No clear ipsilateral shift was observed in the left NPAC in any of these three cases, with WLI values reflecting dominance of the contralateral right hemisphere (see Sup Fig. 3 and Sup Fig. 4). However, the limited dataset prevented us from concluding that cortical reorganization depends on the affected side, as previously suggested (Hanss et al., 2009).

\section{Discussion}

Our results show that UHL induces a brain reorganization that affects auditory processing in the NPAC. This reorganization involves binaural interaction mechanisms and the pattern of cortical activity lateralization. The prolonged alteration of binaural stimulation results in an ipsilateral aural dominance of the BE in the NPAC, a functional reorganization whose extent is correlated with localization abilities. In addition, the disruption of binaural processing predominantly affects the functional integrity of auditory areas in the dorsal stream, reinforcing the hypothesis of a functional dichotomy of the auditory system.

\subsection{Unilateral hearing loss and spatial hearing}

Our results are in line with a large body of evidences showing that unilateral auditory sensory deprivation leads to a degraded integration of binaural auditory inputs and alters spatial hearing (Abel and Lam, 2008; Kumpik and King, 2019; Scheffler et al., 1998; Slattery III \& Middlebrooks, 1994; Vannson et al., 2017). This degradation primarily 


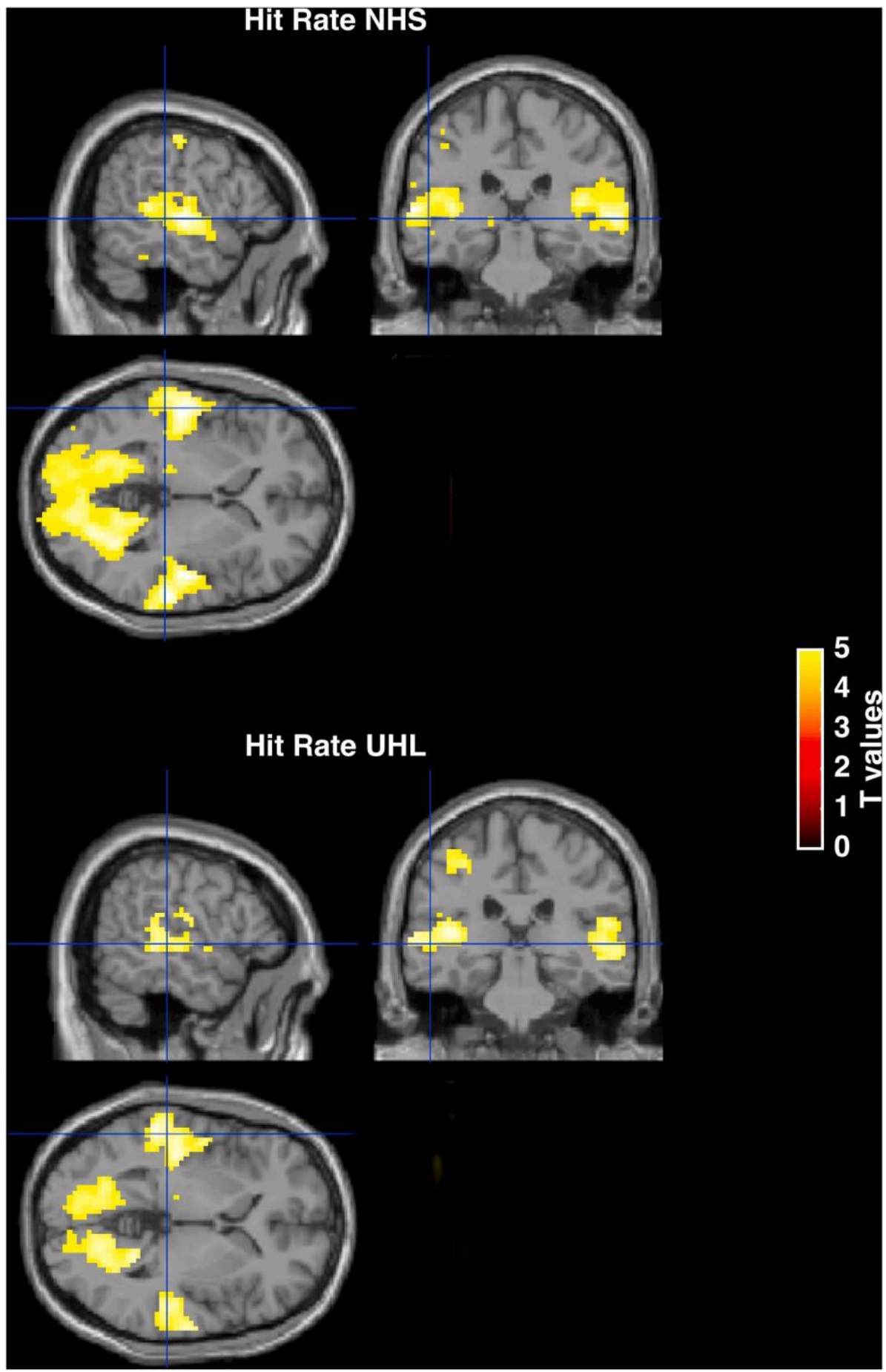

Fig. 7. Regression analysis applied at the wholebrain level between behavioral performances on sound discrimination and brain activity during binaural stimulation. In both NHS (upper panels) and participants with UHL, we observed a similar cortical network whose functional activity was predictive of spatial performances (hit rates; see text). It included the superior and middle temporal gyri and ventral temporal areas. Interestingly, in both cases, the occipital visual areas were also involved. Sagittal, coronal and horizontal views are illustrated at $\mathrm{p}$ uncorrected $=0.001$, for illustration purposes .

affects the binaural auditory cues known as interaural level and time differences (ILD and ITD), which is the first cause for the deficit in sound localization and speech in noise comprehension, as observed in all of our patients. This is clearly reinforced by the observed correlation between hearing thresholds in the deaf ear (PTA) and the spatial hearing (see also Vannson et al., 2017). Furthermore, we showed that the asymmetric binaural inputs lead to abnormal binaural interactions and altered pattern of cortical lateralization. In addition to the alteration of ITD and ILD perception, we suggest that the spatial hearing deficit also relies on the cortical reorganization induced by UHL. Such an assumption is based on the fact that the cortical reorganization is present only in a specific cortical region, the NPAC, not in AI and presumably not at lower stages while the convergence of binaural inputs occurs as early as the brainstem level. In a separate population of patients with UHL, as in other valuable studies (Slattery III \& Middlebrooks, 1994), we observed that a restricted set of patients present near to normal performance for spatial localization (Alzaher et al. in prep). Such adaptation is probably based on the optimized use of monaural spectral cues (Kumpik et al., 2010) and is associated to a pattern of cortical activity different from that observed in hearing controls (Alzaher et al., in prep). These results add further evidence that the specific cortical reorganization induced after long term UHL directly impacts behavioral performances in the spatial domain. 
Table 2

Significant regions from the whole-brain regression analysis between behavioral performances in sound localization and brain activity during binaural stimulation. Clusters revealed by the NHS/UHL participants contrast (in blue in Fig. 8) and located posterior to the PAC (outlined in white) that belongs to the dorsal auditory pathway.

\begin{tabular}{|c|c|c|c|c|c|c|}
\hline Region & $\begin{array}{l}\text { p cluster } \\
\text { (FWE-corr) }\end{array}$ & $\begin{array}{l}\text { Cluster } \\
\text { size }\end{array}$ & $\begin{array}{l}Z \text { - } \\
\text { value }\end{array}$ & $\mathrm{x}$ & y & $\mathrm{z}$ \\
\hline \multirow{3}{*}{$\begin{array}{c}\text { L STG/ } \\
\text { STS, } \\
\text { post }\end{array}$} & 0.00 & 184.00 & 4.14 & -51.00 & -28.00 & -8.00 \\
\hline & & & 4.00 & -48.00 & -16.00 & -8.00 \\
\hline & & & 3.94 & -60.00 & -19.00 & -8.00 \\
\hline \multirow{2}{*}{$\begin{array}{c}\text { R STG/ } \\
\text { STS, } \\
\text { post }\end{array}$} & 0.03 & 119.00 & 3.90 & 63.00 & -25.00 & 4.00 \\
\hline & & & $3: 49$ & $45: 88$ & $\begin{array}{l}-325: 88 \\
-\end{array}$ & $\begin{array}{l}-2.00 \\
10.00\end{array}$ \\
\hline
\end{tabular}

\subsection{Loss of aural preference}

In a normal hearing person, monaural stimulation leads to bilateral activation in both the PAC and NPAC, but with much stronger contralateral activation. This contralateral dominance was confirmed by the present data, in agreement with many other reports in both human (Devlin et al., 2003; Gutschalk and Steinmann, 2015; Schonwiesner et al., 2007) and animal (Clarey et al., 1995; Kelly and Judge, 1994; Phillips and Irvine, 1983) studies. We showed that the cortical contralateral dominance of auditory activity is abolished after UHL, leading to the ipsilateral aural dominance of the BE, in line with previous electrophysiological (Khosla et al., 2003; Li et al., 2012; Po-Hung Li et al., 2003; Ponton et al., 2001) and brain imaging (Burton et al., 2012; Langers et al., 2005; Scheffler et al., 1998) studies. Our first major finding is that these plastic modifications have a cortical origin and are specific to the NPAC. Indeed, Langers et al. (2005) investigated throughout the ascendant auditory pathway all traces of auditory reorganization following a UHL. The authors concluded that UHL consequences (the shift of aural dominance) were only visible at the cortical level irrespective of cytoarchitectonic modifications.

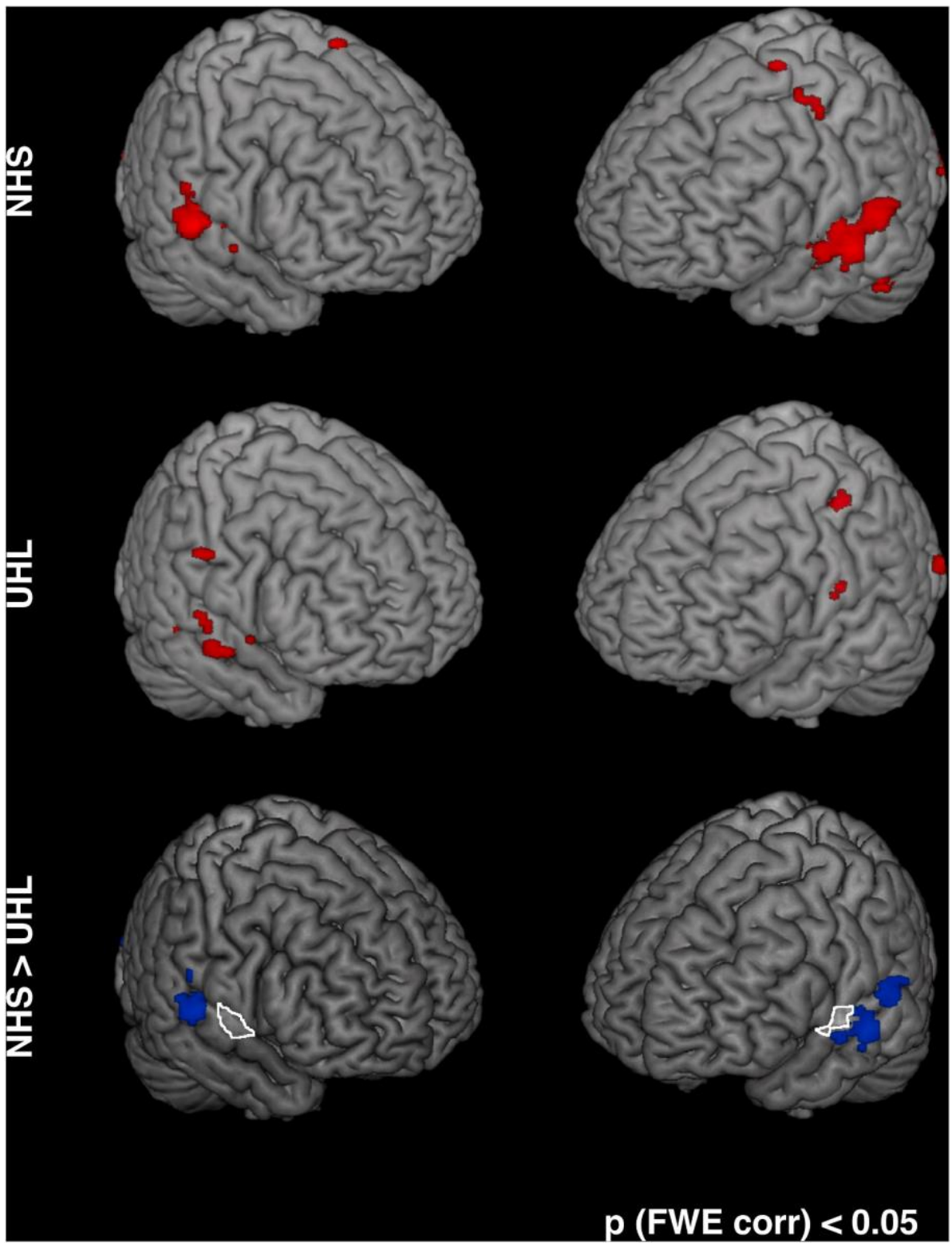

Fig. 8. Regression analysis applied at the wholebrain level between behavioral performances on sound localization and brain activity during binaural stimulation. In NHS (top panel), the activity level in the posterior left and right superior temporal sulcus/gyrus was associated with sound localization performances (RMS errors), whereas in participants with a left UHL (middle panel), only a circumscribed area emerged from the analysis. The NHS/UHL contrast revealed a region (blue) posterior to the PAC (outlined in white) belonging to the dorsal auditory pathway. (For interpretation of the references to color in this figure legend, the reader is referred to the Web version of this article.) 
Contralateral dominance has been reported at the thalamic level for individuals with UHL (Langers et al., 2005) and can be observed in the PAC (present data). This suggests that the ipsilateral dominance observed in the NPAC results from either local mechanisms or corticocortical interactions. In the NHS group, contralateral dominance was less pronounced in the NPAC than in the PAC. This smaller imbalance between the inputs from each ear in the NPAC could explain a greater susceptibility to shift towards the regions ipsilateral to the $\mathrm{BE}$ once contralateral input has decreased owing to UHL. The changes in lateralization reported here correspond to those observed in animal models of congenital UHL (Kral et., 2013a, 2013b; Tillein et al., 2016) or induced (McAlpine et al., 1997; Popelar et al., 1994) with recordings of neuronal activity demonstrating a clear ipsilateral dominance with respect to the $\mathrm{BE}$, reflected in a larger population of ipsilateral responding neurons with an increased excitability and reduced latency. Of interest, animal models of UHL tend to suggest that this reorganization occurs at subcortical levels in the inferior colliculus (McAlpine et al., 1997) and is present in cortical field A1 (Popelar et al., 1994; Tillein et al., 2016), whereas in humans, it is only clearly visible at a higher cortical stage. The origins of these differences are unclear, but they may be due to a stronger functional specialization of human auditory cortical areas. Besides interspecies differences for auditory functions, this discrepancy is probably explained by the fact that most animal studies on hearing loss explore congenital deafness. Indeed, in induced animal neonatal deafness, structural and functional modification are observed from the cochlear nucleus (Syka, 2002) up to the primary auditory cortex (Tillein et al., 2016) with the restriction that the reorganization of the primary auditory cortex is present only if the onset of unilateral hearing loss appears early in development before 2 years of age (Kral, 2013). Furthermore, in animal models, changes in ipsilateral dominance are quite rapid, if not immediate (McAlpine et al., 1997), whereas in humans, after cochlear nerve resection, they can take several months (Bilecen et al., 2000; Burton et al., 2012). Altogether, it is possible that in humans, changes in lateralization rely on gradual cortical interactions and not on the unmasking of existing inputs.

Several reports suggest that left or right monaural deprivation differently impacts cortical reorganization (Burton et al., 2012; Moore et al., 2003), with the assumption that the left hemisphere (right-ear deafness) is more resistant to cortical plasticity (Hanss et al., 2009). Here, most of our results originated from participants with left (poorer ear) UHL, and only a small number of participants with right UHL were included, preventing us from reaching any firm conclusion as to whether side of deafness differently impacts brain reorganization and behavioral performance.

\subsection{Alteration of binaural processing for spatial hearing}

Our second major finding is that there is a strong relationship between the extent of cortical reorganization in the NPAC and the behavioral performances of individuals with UHL. In our study, participants with poorer hearing thresholds performed more poorly on sound localization and had greater ipsilateral lateralization. This indicates that when the plastic reorganization shifts the lateralization of activity towards an ipsilateral dominance, it reduces the ability to locate sounds in a free field. We attribute this relation to the alteration of cortical binaural integration after deafness. At the cortical level, the majority of neurons are spatially selective to contralateral sounds (Barone et al., 1996) and have restricted receptive fields constructed through the excitatory/inhibitory interactions of inputs from each ear (Clarey et al., 1995; Rajan and Irvine, 1996; Samson et al., 1993). The lack of inhibitory inputs from the ipsilateral ear makes the neurons' receptive fields omnidirectional and contributes to a sound localization deficit in monaural conditions (Clarey et al., 1995). We present evidence that this alteration of binaural integration is also present in the human auditory cortex.

In normal hearing persons, binaural stimulation does not correspond to a linear, additive summation of inputs from each ear, but instead to suppression (or occlusion; 37) mechanisms (BII below 1; see Fig. 3). Similar observations of a lower response elicited by binaural stimulation than the sum of monaural stimulations have been made in fMRI studies (Jäncke et al., 2002; Krumbholz et al., 2005), and have been attributed to the suppression of ipsilateral inputs when tested in dichotic situations (Amaral and Langers, 2013; Brancucci et al., 2004; Della Penna et al., 2006; Fujiki et al., 2002). Consequently, the binaural index reveals the presence of excitatory/inhibitory interactions in the PAC and NPAC of hearing humans. UHL profoundly alters binaural integration, reflecting the reduction (in PAC) or absence (in NPAC) of inhibitory inputs from the PE. In animal models of congenital UHL, electrophysiological recordings have provided evidence of altered binaural integration processing (Tillein et al., 2016), resulting in decreased inhibition in the ipsilateral hemisphere and increased inhibition in the hemisphere opposite the BE. Thus, both human and animal studies point to the same conclusion of an alteration of inhibitory interactions following UHL.

\subsection{Consequences of altered spatial hearing on cortical reorganization for auditory rehabilitation}

The main conclusion of our analysis is that UHL induces reorganization of cortical inputs that modifies the functional asymmetry of the auditory cortex, a plastic phenomenon that is deleterious to spatial hearing abilities. In order to assess the potential UHL auditory rehabilitation strategy, the level of cortical asymmetry could be used as an objective marker of brain plasticity. Indeed, within developing children, this plastic reorganization has been shown to circumvent the rehabilitation of the deaf ear with a cochlear implant (Polonenko et al., 2017) and has impacts beyond auditory functions, including on language acquisition (Schaefer et al., 2019). In many cases, however, any alteration of a sensory function is accompanied by intra- or crossmodal compensatory mechanisms, brought about plastic adaptation (Singh et al., 2018). Thus, the impact of UHL on binaural integration may favor processing by the hearing ear (Gordon and Kral, 2019) and produce some perceptual advantages in patients with UHL. These advantages need to be established, although improvements in intensity discrimination (Maslin et al., 2015) or spatial hearing through monaural spectral cues extraction have been reported (Slattery III \& Middlebrooks, 1994).

\subsection{Spatial hearing and dorsal stream in $U H L$}

The regression analysis based on either spatial (sound location) or non-spatial (sound discrimination) performances showed that they are supported by different auditory cortical networks in UHL. Rather than revealing areas that were differently activated by the two tasks, the regression analysis revealed areas whose functional integrity (capacity to respond) could be predictive of behavioral performances.

The areas involved in voice/non-voice discrimination are manifold, as the different acoustic attributes of these two types of stimulus need to be processed in order to form semantic representations. In the participants with UHL, the auditory areas related to their behavioral performances perfectly matched those observed in NHS, and included the lateral parts of the STG, middle temporal gyrus, and ventral temporal areas. This regression highlighted areas of the STS, including the temporal voice area, an auditory area specifically sensitive to the human voice (Belin et al., 2000). In both groups, the involvement of the visual occipital and parietal areas probably reflects the creation of an internal representation when making a decision about semantic sound categorization.

When the analysis was applied to spatial hearing performance, it showed that the cortical network which supports sound localization skills was very circumscribed in participants with UHL compared to that of NHS. In the latter, sound location performance was associated with a small set of auditory areas that mainly encompassed the posterior parts of the STG/STS region, which is known to belong to the dorsal auditory 
stream (Da Costa et al., 2018). Furthermore, the contrast with NHS revealed that this posterior region was functionally absent in participants with UHL, concerning sound localization performances, in line with a study that reported a deficit in sound localization when TMS pulses targeted this posterior region (Ahveninen et al., 2013). Thus, our data clearly demonstrate that the functional integrity of the dorsal stream areas is affected following UHL, which impairs sound localization processing.

These results are of theoretical importance, as they provide additional support for the hypothesis of a functional dichotomy of the auditory cortex, which is now widely accepted for both carnivores and nonhuman primates on anatomical and electrophysiological grounds (Kaas and Hackett, 1998; Lomber and Malhotra, 2008; Rauschecker and Tian, 2000). In humans, a similar dichotomy has been proposed, based on observation of behavioral deficits following cortical lesions (Clarke et al., 2002; Duffour-Nikolov et al., 2012; Zündorf et al., 2016) and brain activation analysis (Arnott et al., 2004; Van der Zwaag et al., 2011). Our study focusing on brain plasticity in UHL provides an original argument in favor of this functional cortical dissociation.

Lastly our results are of interest for the rehabilitation of patients with single-sided deafness treated by a cochlear implant (single-sided deafness-SSD; Arndt et al., 2011; Cabral Junior et al., 2016; Vanderauwera et al., 2020). Indeed, the importance of brain plasticity mechanisms has been shown for a successful hearing rehabilitation (Lazard et al., 2014) and the restoration of a normal aural dominance could be beneficial to an optimal functional recuperation. A handful of studies in developing children (Polonenko et al., 2017; Sharma et al., 2016) and adult (Legris et al., 2018) SSD patients are encouraging and suggest that a normal pattern of cortical activity could be obtained after restoring the binaural auditory inputs using cochlear implantation in the deaf ear.

\section{Conclusion}

The present study revealed that the auditory cortex undergoes major functional reorganization after acquired UHL. Monaural sensory deprivation leads to an increase in the aural dominance of the hearing ear, which impacts mainly on the NPAC and disrupts binaural integration. This plastic reorganization is deleterious to spatial hearing abilities, because it disrupts the functional integrity of the dorsal stream network.

\section{Authors contibutions}

$\mathrm{NV}, \mathrm{KS}, \mathrm{BP}, \mathrm{MM}$ designed the experiment. NV, OD, MM recruited the patients and run the experiments. NV and KS analyzed the data. NV, KS, $\mathrm{CJ}, \mathrm{OD}, \mathrm{BP}, \mathrm{MM}$ wrote and corrected the manuscript.

\section{Fundings}

This study was supported by an ANRT-Cochlear CIFRE PhD funding ( $\mathrm{N}^{\circ} 2012 / 1463$ ) to NV, a grant Agir pour l'audition (APA-RD2015-6B) to $\mathrm{MM}, \mathrm{OD}$ and $\mathrm{BP}$ and the recurrent funding of the CNRS.

\section{Declaration of competing interest}

NV and CJ are Cochlear employees.

\section{Acknowledgements}

The authors are very thankful to all patients and control subjects included in this study. The authors are also grateful to Yohan Gallois, Charles Edouard Molinier, and Gaetan Iversenc for their help in recruiting and testing the patients. The authors would also like to acknowledge Nathalie Vayssiere, Helene Gros-Dagnac and all the technical team of the MRI platform of INSERM U1214 ToNIC for the scanning sessions.

\section{Appendix A. Supplementary data}

Supplementary data to this article can be found online at https://doi. org/10.1016/j.neuropsychologia.2020.107683.

\section{Data availability}

All data are available from the authors upon reasonable request.

\section{References}

Abel, S.M., Lam, K., 2008. Impact of unilateral hearing loss on sound localization. Appl. Acoust. 69 (9), 804-811.

Adriani, M., Bellmann, A., Meuli, R., Fornari, E., Frischknecht, R., Bindschaedler, C., Rivier, F., Thiran, J.-P., Maeder, P., Clarke, S., 2003. Unilateral hemispheric lesions disrupt parallel processing within the contralateral intact hemisphere: an auditory fMRI study. Neuroimage 20, S66-S74.

Ahveninen, J., Huang, S., Nummenmaa, A., Belliveau, J.W., Hung, A.-Y., Jääskeläinen, I. P., Rauschecker, J.P., Rossi, S., Tiitinen, H., Raij, T., 2013. Evidence for distinct human auditory cortex regions for sound location versus identity processing. Nat. Commun. 4, 2585. https://doi.org/10.1038/ncomms3585.

Amaral, A.A., Langers, D.R., 2013. The relevance of task-irrelevant sounds: hemispheric lateralization and interactions with task-relevant streams. Front. Neurosci. 7, 264.

Arndt, S., Aschendorff, A., Laszig, R., Beck, R., Schild, C., Kroeger, S., Ihorst, G., Wesarg, T., 2011. Comparison of pseudobinaural hearing to real binaural hearing rehabilitation after cochlear implantation in patients with unilateral deafness and tinnitus. Otol. Neurotol. 32 (1), 39-47.

Arnott, S.R., Binns, M.A., Grady, C.L., Alain, C., 2004. Assessing the auditory dualpathway model in humans. Neuroimage 22 (1), 401-408.

Barone, P., Clarey, J.C., Irons, W.A., Imig, T.J., 1996. Cortical synthesis of azimuthsensitive single-unit responses with nonmonotonic level tuning: a thalamocortical comparison in the cat. J. Neurophysiol. 75 (3), 1206-1220.

Belin, P., Zatorre, R.J., Hoge, R., Evans, A.C., Pike, B., 1999. Event-related fMRI of the auditory cortex. Neuroimage 10 (4), 417-429.

Belin, P., Zatorre, R.J., Lafaille, P., Ahad, P., Pike, B., 2000. Voice-selective areas in human auditory cortex. Nature 403 (6767), 309.

Bilecen, D., Seifritz, E., Radü, E.W., Schmid, N., Wetzel, S., Probst, R., Scheffler, K., 2000. Cortical reorganization after acute unilateral hearing loss traced by fMRI. Neurology 54 (3), 765,765 .

Brancucci, A., Babiloni, C., Babiloni, F., Galderisi, S., Mucci, A., Tecchio, F., Zappasodi, F., Pizzella, V., Romani, G.L., Rossini, P.M., 2004. Inhibition of auditory cortical responses to ipsilateral stimuli during dichotic listening: evidence from magnetoencephalography. Eur. J. Neurosci. 19 (8), 2329-2336.

Bronkhorst, A.W., Plomp, R., 1989. Binaural speech intelligibility in noise for hearing impaired listeners. J. Acoust. Soc. Am. 86 (4), 1374-1383.

Burton, H., Firszt, J.B., Holden, T., Agato, A., Uchanski, R.M., 2012. Activation lateralization in human core, belt, and parabelt auditory fields with unilateral deafness compared to normal hearing. Brain Res. 1454, 33-47.

Cabral Junior, F., Pinna, M.H., Alves, R.D., Malerbi, A.F., dos, S., Bento, R.F., 2016. Cochlear implantation and single-sided deafness: a systematic review of the literature. Int. Arch. Otorhinolaryngol. 20 (1), 69-75.

Carpenter, J., Bithell, J., 2000. Bootstrap confidence intervals: when, which, what? A practical guide for medical statisticians. Stat. Med. 19 (9), 1141-1164.

Clarey, J.C., Barone, P., Irons, W.A., Samson, F.K., Imig, T.J., 1995. Comparison of noise and tone azimuth tuning of neurons in cat primary auditory cortex and medical geniculate body. J. Neurophysiol. 74 (3), 961-980.

Clarke, S., Thiran, A.B., Maeder, P., Adriani, M., Vernet, O., Regli, L., Cuisenaire, O., Thiran, J.-P., 2002. What and where in human audition: selective deficits following focal hemispheric lesions. Exp. Brain Res. 147 (1), 8-15.

Da Costa, S., Clarke, S., Crottaz-Herbette, S., 2018. Keeping track of sound objects in space: the contribution of early-stage auditory areas. Hear. Res. 366, 17-31.

Della Penna, S., Brancucci, A., Babiloni, C., Franciotti, R., Pizzella, V., Rossi, D. Torquati, K., Rossini, P.M., Romani, G.L., 2006. Lateralization of dichotic speech stimuli is based on specific auditory pathway interactions: neuromagnetic evidence. Cerebr. Cortex 17 (10), 2303-2311.

Devlin, J.T., Raley, J., Tunbridge, E., Lanary, K., Floyer-Lea, A., Narain, C., Cohen, I. Behrens, T., Jezzard, P., Matthews, P.M., 2003. Functional asymmetry for auditory processing in human primary auditory cortex. J. Neurosci. 23 (37), 11516-11522.

Duffour-Nikolov, C., Tardif, E., Maeder, P., Thiran, A.B., Bloch, J., Frischknecht, R. Clarke, S., 2012. Auditory spatial deficits following hemispheric lesions: dissociation of explicit and implicit processing. Neuropsychol. Rehabil. 22 (5), 674-696.

Eickhoff, S.B., Stephan, K.E., Mohlberg, H., Grefkes, C., Fink, G.R., Amunts, K., Zilles, K. 2005. A new SPM toolbox for combining probabilistic cytoarchitectonic maps and functional imaging data. Neuroimage 25 (4), 1325-1335.

Fujiki, N., Jousmäki, V., Hari, R., 2002. Neuromagnetic responses to frequency-tagged sounds: a new method to follow inputs from each ear to the human auditory cortex during binaural hearing. J. Neurosci. 22 (3), RC205. RC205.

Gordon, K., Kral, A., 2019. Animal and human studies on developmental monaural hearing loss. Hear. Res. 380, 60-74.

Grothe, B., Pecka, M., McAlpine, D., 2010. Mechanisms of sound localization in mammals. Physiol. Rev. 90 (3), 983-1012.

Gutschalk, A., Steinmann, I., 2015. Stimulus dependence of contralateral dominance in human auditory cortex. Hum. Brain Mapp. 36 (3), 883-896. 
Hanss, J., Veuillet, E., Adjout, K., Besle, J., Collet, L., Thai-Van, H., 2009. The effect of long-term unilateral deafness on the activation pattern in the auditory cortices of French-native speakers: influence of deafness side. BMC Neurosci. 10 (1), 23.

Heinrich, A., Mikkola, T.M., Polku, H., Törmäkangas, T., Viljanen, A., 2019. Hearing in real-life environments (HERE): structure and reliability of a questionnaire on perceived hearing for older adults. Ear Hear. 40 (2), 368-380.

Jamison, H.L., Watkins, K.E., Bishop, D.V., Matthews, P.M., 2006. Hemispheric specialization for processing auditory nonspeech stimuli. Cerebr. Cortex 16 (9), $1266-1275$

Jäncke, L., Wüstenberg, T., Scheich, H., Heinze, H.-J., 2002. Phonetic perception and the temporal cortex. Neuroimage 15 (4), 733-746. https://doi.org/10.1006/ nimg.2001.1027.

Jansen, S., Luts, H., Wagener, K.C., Kollmeier, B., Del Rio, M., Dauman, R., James, C. Fraysse, B., Vormès, E., Frachet, B., 2012. Comparison of three types of French speech-in-noise tests: a multi-center study. Int. J. Audiol. 51 (3), 164-173.

Kaas, J.H., Hackett, T.A., 1998. Subdivisions of AuditoryCortex and levels of processing in primates. Audiology and Neurotology 3 (2-3), 73-85. https://doi.org/10.1159/ 000013783.

Kelly, J.B., Judge, P.W., 1994. Binaural organization of primary auditory cortex in the ferret (Mustela putorius). J. Neurophysiol. 71 (3), 904-913.

Khosla, D., Ponton, C.W., Eggermont, J.J., Kwong, B., Dort, M., Vasama, J.-P., 2003 Differential ear effects of profound unilateral deafness on the adult human central auditory system. Journal of the Association for Research in Otolaryngology 4 (2), 235-249.

Kral, A., 2013. Auditory critical periods: a review from system's perspective. Neuroscience $247,117-133$.

Kral, A., Land, R., Baumhoff, P., Tillein, J., Hubka, P., Lomber, S.G., 2013. Cross-modal plasticity in the congenitally deaf cat. Multisensory Res. 26, 37, 37.

Kral, Andrej, Hubka, P., Heid, S., Tillein, J., 2013. Single-sided deafness leads to unilateral aural preference within an early sensitive period. Brain 136 (1), 180-193.

Krumbholz, K., Schönwiesner, M., Rübsamen, R., Zilles, K., Fink, G.R., Von Cramon, D.Y. 2005. Hierarchical processing of sound location and motion in the human brainstem and planum temporale. Eur. J. Neurosci. 21 (1), 230-238.

Kumpik, D.P., Kacelnik, O., King, A.J., 2010. Adaptive reweighting of auditory localization cues in response to chronic unilateral earplugging in human J. Neurosci. 30 (14), 4883-4894.

Kumpik, D.P., King, A.J., 2019. A review of the effects of unilateral hearing loss on spatial hearing. Hear. Res. 372, 17-28.

Langers, D.R., van Dijk, P., Backes, W.H., 2005. Lateralization, connectivity and plasticity in the human central auditory system. Neuroimage 28 (2), 490-499.

Lazard, D.S., Innes-Brown, H., Barone, P., 2014. Adaptation of the communicative brain to post-lingual deafness. Evidence from functional imaging. Hear. Res. 307, 136143.

Legris, E., Galvin, J., Roux, S., Gomot, M., Aoustin, J.-M., Marx, M., He, S., Bakhos, D., 2018. Cortical reorganization after cochlear implantation for adults with singlesided deafness. PloS One 13 (9), e0204402.

Li, L.P.-H., Shiao, A.-S., Chen, K.-C., Lee, P.-L., Niddam, D.M., Chang, S.-Y., Hsieh, J.-C. 2012. Neuromagnetic index of hemispheric asymmetry prognosticating the outcome of sudden hearing loss. PloS One 7 (4), e35055.

Lomber, S.G., Malhotra, S., 2008. Double dissociation of'what'and'where'processing in auditory cortex. Nat. Neurosci. 11 (5), 609.

Maslin, M.R., Taylor, M., Plack, C.J., Munro, K.J., 2015. Enhanced intensity discrimination in the intact ear of adults with unilateral deafness. J. Acoust. Soc. Am. 137 (6), EL408-EL414.

Massida, Z., Belin, P., James, C., Rouger, J., Fraysse, B., Barone, P., Deguine, O., 2011. Voice discrimination in cochlear-implanted deaf subjects. Hear. Res. 275 (1-2), $120-129$.

McAlpine, D., Martin, R.L., Mossop, J.E., Moore, D.R., 1997. Response properties of neurons in the inferior colliculus of the monaurally deafened ferret to acoustic stimulation of the intact ear. J. Neurophysiol. 78 (2), 767-779.

Moore, D.R., Hartley, D.E., Hogan, S. C., 2003. Effects of otitis media with effusion (OME) on central auditory function. Int. J. Pediatr. Otorhinolaryngol. 67, S63-S67.

Morosan, P., Rademacher, J., Palomero-Gallagher, N., Zilles, K., 2005. Anatomica organization of the human auditory cortex: cytoarchitecture and transmitter receptors. The Auditory Cortex. Psychology Press, pp, 45-68.

Morosan, P., Rademacher, J., Schleicher, A., Amunts, K., Schormann, T., Zilles, K., 2001. Human primary auditory cortex: cytoarchitectonic subdivisions and mapping into spatial reference system. Neuroimage 13 (4), 684-701.

Oldfield, R.C., 1971. The assessment and analysis of handedness: the Edinburgh inventory. Neuropsychologia 9 (1), 97-113.

Phillips, D.P., Irvine, D.R., 1983. Some features of binaural input to single neurons in physiologically defined area AI of cat cerebral cortex. J. Neurophysiol. 49 (2), 383395

Po-Hung Li, L., Shiao, A.-S., Lin, Y.-Y., Chen, L.-F., Niddam, D.M., Chang, S.-Y., Lien, C. F., Chou, N.-S., Ho, L.-T., Hsieh, J.-C., 2003. Healthy-side dominance of cortical neuromagnetic responses in sudden hearing loss. Ann. Neurol. 53 (6), 810-815.

Polonenko, M.J., Gordon, K.A., Cushing, S.L., Papsin, B.C., 2017. Cortical organization restored by cochlear implantation in young children with single sided deafness. Sci. Rep. 7 (1), 16900.
Ponton, C.W., Vasama, J.-P., Tremblay, K., Khosla, D., Kwong, B., Don, M., 2001. Plasticity in the adult human central auditory system: evidence from late-onset profound unilateral deafness. Hear. Res. 154 (1-2), 32-44.

Popelar, J., Erre, J.-P., Aran, J.-M., Cazals, Y., 1994. Plastic changes in ipsi-contralateral differences of auditory cortex and inferior colliculus evoked potentials after injury to one ear in the adult Guinea pig. Hear. Res. 72 (1-2), 125-134.

Rajan, R., Irvine, D.R., 1996. Features of, and boundary conditions for, lesion-induced reorganization of adult auditory cortical maps. Auditory System Plasticity and Regeneration 224-237.

Rauschecker, J.P., Scott, S.K., 2009. Maps and streams in the auditory cortex: nonhuman primates illuminate human speech processing. Nat. Neurosci. 12 (6), 718.

Rauschecker, J.P., Tian, B., 2000. Mechanisms and streams for processing of "what" and "where" in auditory cortex. Proc. Natl. Acad. Sci. Unit. States Am. 97 (22), 1180011806

Sadoun, A., Chauhan, T., Mameri, S., Zhang, Y.F., Barone, P., Deguine, O., Strelnikov, K., 2020. Stimulus-specific information is represented as local activity patterns across the brain. Neuroimage 223, 117326. https://doi.org/10.1016/j. neuroimage.2020.117326.

Samson, F. K., Clarey, J.C., Barone, P., Imig, T.J., 1993. Effects of ear plugging on singleunit azimuth sensitivity in cat primary auditory cortex. I. Evidence for monaural directional cues. J. Neurophysiol. 70 (2), 492-511.

Schaefer, S.M., de Kruijf, M., Henderson, L., Metryka, A., O’Driscoll, M., Bruce, I.A., 2019. Improved speech and language development after unilateral cochlear implantation in children with a potentially useable contralateral ear. Cochlear Implants Int. 20 (1), 39-46.

Scheffler, K., Bilecen, D., Schmid, N., Tschopp, K., Seelig, J., 1998. Auditory cortical responses in hearing subjects and unilateral deaf patients as detected by functional magnetic resonance imaging. New York, NY: 1991 Cerebr. Cortex 8 (2), 156-163.

Schonwiesner, M., Novitski, N., Pakarinen, S., Carlson, S., Tervaniemi, M., Naatanen, R., 2007. Heschl's gyrus, posterior superior temporal gyrus, and mid-ventrolateral prefrontal cortex have different roles in the detection of acoustic changes. J. Neurophysiol. 97 (3), 2075-2082.

Sharma, A., Glick, H., Campbell, J., Torres, J., Dorman, M., Zeitler, D.M., 2016. Cortical plasticity and Re-organization in pediatric single-sided deafness pre-and postcochlear implantation: a case study. Otology \& neurotology: Official Publication of the American Otological Society, American Neurotology Society [and] European Academy of Otology and Neurotology 37 (2), e26.

Singh, A.K., Phillips, F., Merabet, L.B., Sinha, P., 2018. Why does the cortex reor ganize after sensory loss? Trends Cognit. Sci. 22 (7), 569-582.

Slattery III, W.H., Middlebrooks, J.C., 1994. Monaural sound localization: acute versus chronic unilateral impairment. Hear. Res. 75 (1-2), 38-46.

Stefanatos, G.A., Joe, W.Q., Aguirre, G.K., Detre, J.A., Wetmore, G., 2008. Activation of human auditory cortex during speech perception: effects of monaural, binaural, and dichotic presentation. Neuropsychologia 46 (1), 301-315.

Suzuki, M., Kouzaki, H., Nishida, Y., Shiino, A., Ito, R., Kitano, H., 2002. Cortical representation of hearing restoration in patients with sudden deafness. Neuroreport 13 (14), 1829-1832.

Syka, J., 2002. Plastic changes in the central auditory system after hearing loss, restoration of function, and during learning. Physiol. Rev. 82 (3), 601-636.

Tillein, J., Hubka, P., Kral, A., 2016. Monaural congenital deafness affects aura dominance and degrades binaural processing. Cerebr. Cortex 26 (4), 1762-1777.

van der Heijden, K., Rauschecker, J.P., de Gelder, B., Formisano, E., 2019. Cortical mechanisms of spatial hearing. Nat. Rev. Neurosci. 20 (10), 609-623.

van der Heijden, K., Rauschecker, J.P., Formisano, E., Valente, G., de Gelder, B., 2018. Active sound localization sharpens spatial tuning in human primary auditory cortex. J. Neurosci. 38 (40), 8574-8587.

Van der Zwaag, W., Gentile, G., Gruetter, R., Spierer, L., Clarke, S., 2011. Where sound position influences sound object representations: a 7-T fMRI study. Neuroimage 54 (3), 1803-1811.

Van Wanrooij, M.M., Van Opstal, A.J., 2007. Sound localization under perturbed binaural hearing. J. Neurophysiol. 97 (1), 715-726.

Vanderauwera, J., Hellemans, E., Verhaert, N., 2020. Research insights on neural effects of auditory deprivation and restoration in unilateral hearing loss: a systematic review. J. Clin. Med. 9 (3), 812.

Vannson, N. James, C., Fraysse, B., Strelnikov, K., Barone, P., Deguine, O, Marx, M. 2015. Quality of life and auditory performance in adults with asymmetric hearing loss. Audiology and Neurotology 20 (Suppl. 1), 38-43.

Vannson, N., James, C.J., Fraysse, B., Lescure, B., Strelnikov, K., Deguine, O., Barone, P. Marx, M., 2017. Speech-in-noise perception in unilateral hearing loss: relation to puretone thresholds and brainstem plasticity. Neuropsychologia 102, 135-143.

Vasama, J.-P., Mäkelä, J.P., 1995. Auditory pathway plasticity in adult humans after unilateral idiopathic sudden sensorineural hearing loss. Hear. Res. 87 (1-2), 132 140

Weeks, R., Horwitz, B., Aziz-Sultan, A., Tian, B., Wessinger, C.M., Cohen, L.G., Hallett, M., Rauschecker, J.P., 2000. A positron emission tomographic study of auditory localization in the congenitally blind. J. Neurosci. 20 (7), 2664-2672.

Zündorf, I.C., Lewald, J., Karnath, H.-O., 2016. Testing the dual-pathway model for auditory processing in human cortex. Neuroimage 124, 672-681. 